\title{
G-Protein-Coupled Receptor Kinase-5 Mediates Inflammation but Does Not Regulate Cellular Infiltration or Bacterial Load in a Polymicrobial Sepsis Model in Mice
}

\author{
Nandakumar Packiriswamy ${ }^{b}$ Taehyung Lee ${ }^{a}$ Pongali B. Raghavendra ${ }^{a}$ \\ Haritha Durairaj $^{\mathrm{a}}$ Hongbing Wang $^{\mathrm{a}}$ Narayanan Parameswaran ${ }^{\mathrm{a}, \mathrm{b}}$ \\ ${ }^{a}$ Division of Human Pathology, Department of Physiology, and ${ }^{b}$ Comparative Medicine and Integrative Biology \\ Program, College of Veterinary Medicine, Michigan State University, East Lansing, Mich., USA
}

\section{Key Words}

Apoptosis · Cytokines · Host defense - Immune response $\cdot$

Macrophages · Sepsis · Septic shock · Toll-like receptor

\begin{abstract}
NFKB-dependent signaling is an important modulator of inflammation in several diseases including sepsis. G-proteincoupled receptor kinase-5 (GRK5) is an evolutionarily conserved regulator of the NFKB pathway. We hypothesized that GRK5 via NFKB regulation plays an important role in the pathogenesis of sepsis. To test this we utilized a clinically relevant polymicrobial sepsis model in mice that were deficient in GRK5. We subjected wild-type (WT) and GRK5 knockout (KO) mice to cecal ligation and puncture (CLP)-induced polymicrobial sepsis and assessed the various events in sepsis pathogenesis. CLP induced a significant inflammatory response in the WT and this was markedly attenuated in the $\mathrm{KO}$ mice. To determine the signaling mechanisms and the role of NFKB activation in sepsis-induced inflammation, we assessed the levels of $\mathrm{IKBa}$ phosphorylation and expression of NFKB-dependent genes in the liver in the two genotypes. Both IKBa phosphorylation and gene expression were significantly inhibited in the GRK5 KO compared to the WT mice. Interestingly, however, GRK5 did not modulate either immune cell infiltration (to the primary site of infection) or
\end{abstract}

local/systemic bacterial load subsequent to sepsis induction. In contrast GRK5 deficiency significantly inhibited sepsis-induced plasma corticosterone levels and the consequent thymocyte apoptosis in vivo. Associated with these outcomes, CLP-induced mortality was significantly prevented in the GRK5 KO mice in the presence of antibiotics. Together, our studies demonstrate that GRK5 is an important regulator of inflammation and thymic apoptosis in polymicrobial sepsis and implicate GRK5 as a potential molecular target in sepsis.

Copyright $\odot 2013$ S. Karger AG, Basel

\section{Introduction}

G-protein-coupled receptor kinases (GRKs) are serine/ threonine kinases well known for their role in phosphorylation of G-protein-coupled receptors [1]. Functionally GRKs have been linked to a number of cell signaling processes, not only related to their role in G-protein-coupled receptor phosphorylation, but also in their ability to phosphorylate or scaffold a number of intracellular signaling proteins [2]. GRKs are functionally grouped into three classes: GRK1-like (GRK1 and GRK7, otherwise known as rhodopsin kinases), GRK2-like [GRK2 and GRK3, otherwise known as $\beta$ ARK1 and 2 ( $\beta$-adrenergic receptor kinases 1 and 2)] and GRK4-like (GRK4, GRK5 and GRK6).

\section{KARGER}

E-Mail karger@karger.com

www.karger.com/jin
(C) 2013 S. Karger AG, Basel

$1662-811 \mathrm{X} / 13 / 0054-0401 \$ 38.00 / 0$
Prof. Narayanan Parameswaran

Division of Human Pathology, Department of Physiology, Michigan State University, \#2201 Biomedical Physical Sciences Building

567 Wilson Road, East Lansing, MI 48824 (USA)

E-Mail paramesw@msu.edu 
Even though there is some specificity in terms of their tissue distribution, GRKs especially GRK2 and GRK5 are ubiquitously expressed in many cell types including immune cells [2].

Of the seven members of the GRK family, GRK5 was first identified as a kinase that phosphorylates $\beta_{2^{-}}$ adrenergic receptor, $\mathrm{m} 2$ muscarinic cholinergic receptor, and rhodopsin [3]. Using GRK5-deficient mice, Gainetdinov et al. [4] further identified a critical role for GRK5 in muscarinic receptor signaling in vivo. Subsequently, however, several studies have demonstrated a broad role for this kinase in cell signaling. For example, GRK5 has been shown to phosphorylate and/or interact with nonreceptor substrates including arrestin-2 [5], F-actin [6], HDAC5 [7], Hip [8], IкBa [9], p105 [10], Lrp6 [11], nucleophosmin [12] and p53 [13]. Based on its role in cell signaling, GRK5 has been proposed to be a critical kinase in the pathogenesis of several diseases including endotoxemia [14], cancer [15], Alzheimer's [16] and atherosclerosis [17]. In addition, GRK5 levels are modulated in a number of diseases including sepsis, heart failure, obesity, cystic fibrosis, cancer and mental disorders [2]. We have recently demonstrated that the GRK5 knockout (KO) mice have attenuated the ability to produce cytokines in vivo in response to lipopolysaccharide (LPS) [a Toll-like receptor 4 (TLR4) ligand] [14]. We further showed GRK5 to be an important regulator of signaling from multiple Toll-like receptor ligands including TLR2 and TLR3 in vivo [18]. Interestingly, a recent study also demonstrated that GRK5 is a critical mediator of inflammation in drosophila and zebra fish models [19]. However, the role of GRK5 in the pathogenesis of a clinically relevant model of polymicrobial sepsis is not known.

Sepsis is the leading cause of death among intensive care patients [20]. Dysregulated inflammatory response is a prominent modulator of sepsis progression, causing coagulation derangements, apoptosis of lymphoid and nonlymphoid tissues and organ dysfunction [21]. Despite the improvements in resuscitation and antibiotic supportive care, the high incidence and fatality in sepsis underscore the need for better understanding of the pathophysiology of sepsis and to identify new molecular therapeutic targets. Using a clinically relevant polymicrobial sepsis model [22], we demonstrate here that GRK5 is an important modulator of sepsis progression, inflammation, thymocyte apoptosis and mortality. We further demonstrate that GRK5 is an important regulator of sepsis-induced NFKB activation in the liver. Together, our studies implicate GRK5 as an important molecular target in the pathogenesis of polymicrobial sepsis.

\section{Materials and Methods}

\section{Materials}

Protease inhibitor cocktail tablets were from Roche Applied Science (Indianapolis, Ind., USA); pIkBa, pERK1/2, pP38 and pJNK and tubulin antibodies were from Cell Signaling Technology, Inc. (Danvers, Mass., USA) and Sigma (St. Louis, Mo., USA), respectively. Ultrapure Escherichia coli (0111:B4) LPS was from InvivoGen (San Diego, Calif., USA) and dexamethasone was from Sigma.

Mice

GRK5 KO mice were obtained from Jackson Labs and have been previously described [14]. Animals used for experiments were 8- to 12 -week-old males. They were housed 4-5 mice per cage at $22-24^{\circ} \mathrm{C}$ with $50 \%$ humidity and a 12 -hour light-dark cycle. All animal procedures were approved by Michigan State University Animal Care and Use Committee.

\section{Sepsis Model}

Polymicrobial intra-abdominal sepsis was induced by the cecal ligation and puncture (CLP) technique [23]. Briefly, mice were anesthetized by administering ketamine $(80 \mathrm{mg} / \mathrm{kg})$ and xylazine $(5 \mathrm{mg} / \mathrm{kg})$ intraperitoneally. The cecum was exteriorized, ligated and punctured twice with a 20 -gauge needle. Sham surgeries were carried out to serve as control in which the exteriorized cecum was neither ligated nor punctured. All animals were administered subcutaneously with $1 \mathrm{ml}$ of warm saline after surgery. In one set of survival experiments, antibiotics (ceftriaxone $25 \mathrm{mg} / \mathrm{kg}$ and metronidazole $15 \mathrm{mg} / \mathrm{kg}$ ) were administered (intraperitoneally) $1 \mathrm{~h}$ postsepsis and every $24 \mathrm{~h}$ for 5 days.

\section{Peritoneal Lavage Fluid and Blood Collection}

Peritoneal exudate fluid was collected at different time points postsepsis as described before [24]. Briefly, the peritoneal cavity was lavaged with $7 \mathrm{ml}$ of RPMI media with $10 \%$ FBS and peritoneal fluid was collected, centrifuged to separate the cells and the supernatants stored at $-80^{\circ} \mathrm{C}$ until further analysis. Blood was collected by cardiac puncture and plasma was separated (by centrifugation) and stored at $-80^{\circ} \mathrm{C}$ until further analysis.

\section{Cytokine/Chemokine Measurements}

Cytokines and chemokines were measured from peritoneal exudate and plasma using ELISA kits from eBiosciences, Inc. as described before [25].

\section{Bacterial Counts}

Bacterial load was determined in blood and peritoneal fluid at different time points as described before [24]. Briefly, blood and peritoneal fluid were serially diluted and plated on Trypticase ${ }^{\mathrm{TM}}$ Soy Agar with 5\% Sheep Blood (BD Biosciences) and incubated at $37^{\circ} \mathrm{C}$ for $48 \mathrm{~h}$. Colony-forming units were counted to determine bacterial load and expressed as colony-forming units per milliliter.

\section{Determination of Thymic Cell Numbers and Apoptosis}

Thymi were collected from septic and sham-operated mice 20 and $36 \mathrm{~h}$ postsepsis and single cell suspension was prepared as described before [26]. For determining cell number changes, cells were counted using a hemocytometer. Cells were also labeled with 
Annexin V and propidium iodide (PI) [following the manufacturer's instructions (eBiosciences)] to determine the apoptotic cells by flow cytometry (LSRII, BD Biosciences) as described before [27]. In addition, cells were labeled with anti-CD4-PE-Cy7 and anti-CD8-PE for determining CD4+ and CD8+ cells in the thymus and data were acquired using LSRII (BD Biosciences) and analyzed using Flowjo software (Tree Star, Inc., Ashland, Oreg., USA).

\section{Caspase Activity Assays}

Thymocytes (obtained as described above) were lysed in buffer (50 mM HEPES, 0.1\% CHAPS, 1 mM DTT, $0.1 \mathrm{mM}$ EDTA and $0.4 \%$ Triton X-100, pH 7.4) at $4^{\circ} \mathrm{C}$ for $15 \mathrm{~min}$. The cell lysate was collected and the protein content determined (Bio-Rad). $10 \mu \mathrm{g}$ of the cell lysate was incubated with the fluorescent substrates (Ac-DEVDAFC, Z-IETD-AFC and Ac-LEHD-AFC) to determine caspase-3, 8 and 9 activities at $100 \mu \mathrm{M}$ in the assay buffer (50 mM HEPES, $1 \%$ sucrose, $0.1 \%$ CHAPS and $10 \mathrm{mM}$ DTT, pH 7.4) as described [28]. The fluorescence of the cleaved substrates was determined spectrofluorometrically (excitation of $400 \mathrm{~nm}$ and emission of $505 \mathrm{~nm}$ ) in Tecan Spectra FluorPlus fluorescence plate reader. Data are presented as picograms of cleaved AFC per milligram protein per minute calculated from a standard curve plot with free AFC.

\section{In vitro Stimulation of Septic Peritoneal Cells}

Peritoneal cells collected from wild-type (WT) and GRK5 KO mice $36 \mathrm{~h}$ postsepsis were washed with PBS and plated in 12-well plates at 1 million cells/well. Cells were then stimulated (or not) with LPS $(0.5 \mu \mathrm{g} / \mathrm{ml})$ for $12 \mathrm{~h}$ and supernatants collected and assayed for the indicated cytokines by ELISA.

\section{Restraint Stress}

Eight- to 10-week-old GRK5 WT and KO mice were subjected to stress with physical restraint as previously described [29]. Briefly, mice were placed in a $50-\mathrm{ml}$ centrifuge tube with multiple openings for ventilation and held horizontally for $30 \mathrm{~min}$. After the stipulated time, blood was collected for corticosterone measurement.

\section{Corticosterone Measurements}

Plasma corticosterone levels were measured using a corticosterone EIA kit from Cayman Chemical (Ann Arbor, Mich., USA) according to the manufacturer's instructions.

\section{RNA Extraction and Real-Time Q-PCR}

Liver and lung samples from septic and sham-operated mice were collected $12 \mathrm{~h}$ post-CLP, and total RNA was extracted using the Qiagen's RNeasy Mini kit. Reverse transcription was carried out with $1 \mu \mathrm{g}$ of RNA with the Promega cDNA synthesis kit. Realtime Q-PCR was performed as described before for the expression of I $\mathrm{B} \alpha \boldsymbol{\alpha}, \mathrm{IL}-6, \mathrm{IL}-1 \beta$ and HPRT [9]. Primers were obtained from IDT DNA Technologies. The following primers were used: I $\mathrm{B} \alpha$ forward: TGG CCA GTG TAG CAG TCT TG, reverse: GAC ACG TGT GGC CAT TGT AG; IL-6 forward: ACA AGT CGG AGG CTT AAT TAC ACA T, reverse: TTG CCA TTG CAC AAC TCT TTT C; IL- $1 \beta$ forward: TCG CTC AGG GTC ACA AGA AA, reverse: CAT CAG AGG CAA GGA GGA AAA C, and HPRT forward: AAG CCT AAG ATG AGC GCA AG, reverse: TTA CTA GGC AGA TGG CCA CA. Real-time Q-PCR was performed using ABI Fast 7500 (Applied Biosystems) and all the genes were normalized to HPRT.

GRK5 Mediates Sepsis-Induced Inflammation

\section{Western Blot Analysis}

Cytoplasmic extracts from frozen liver tissue samples were prepared by homogenizing the tissue in lysis buffer $(1 \mathrm{M}$ HEPES, $2 \mathrm{M} \mathrm{KCl}, 0.5 \mathrm{M}$ EDTA and $0.1 \mathrm{M}$ EGTA along with protease and phosphatase inhibitors). The protein concentrations in the extracts were determined and equivalent amounts of protein were loaded onto the gels for Western blot analysis. Immunoblotting was carried out for $\mathrm{pI \kappa Ba}, \mathrm{pERK}, \mathrm{pJNK}, \mathrm{pp} 38$ and tubulin as described before [14]. The bands were quantified using the image-J (for chemiluminescence) or Licor's Odyssey program (for fluorescence).

\section{Statistical Analysis}

All data are presented as the mean \pm SEM. Two group comparisons were performed using Student's t test and comparisons of more than two groups were done by ANOVA with the post-Bonferroni test. Survival studies were analyzed by log-rank test (Mantel-Cox) [30-32] as well as by factorial analysis. All statistical analyses (except factorial analysis) were performed using GraphPad Prism Software (San Diego, Calif., USA) and $p<0.05$ were considered statistically significant. Factorial analysis for survival [two genotypes (WT and $\mathrm{KO}$ ) and two treatments (without and with antibiotics)] was performed in consultation with the Center for Statistical Training and Consulting of the Michigan State University and using the SPSS software program.

\section{Results}

In previous studies, we demonstrated that the GRK5deficient mice have an attenuated inflammatory response after in vivo stimulation with TLR ligands $[14,18]$. Given that the progression of polymicrobial sepsis is in part dependent on the stimulation of multiple TLRs, we examined the role of GRK5 in a clinically relevant model of polymicrobial sepsis. For this, we subjected WT and GRK5 KO mice to CLP and determined the various pathogenic events including inflammatory response, immune cell infiltration, thymic apoptosis, bacterial load and mortality.

\section{GRK5 Mediates Sepsis-Induced Cytokine Production}

Cytokine and chemokine levels were examined in the peritoneal fluid and plasma from the different groups of mice (sham and CLP in the WT and KO groups) at $12 \mathrm{~h}$ after surgery. As shown in figure 1a, IL-6, IL-10, TNFa and MCP-1 levels in the peritoneal fluid were significantly decreased in the KO septic compared to the WT septic mice. Peritoneal IL-12/23 (total p40) was also inhibited in the $\mathrm{KO}$ mice but did not reach statistical significance. Similar to the peritoneal fluid, plasma levels of IL- 6 and IL-10 were significantly decreased in the KO compared to the WT septic mice (fig. 1b). Plasma levels of TNFa, IL-12/23 and MCP-1 did not significantly dif- 


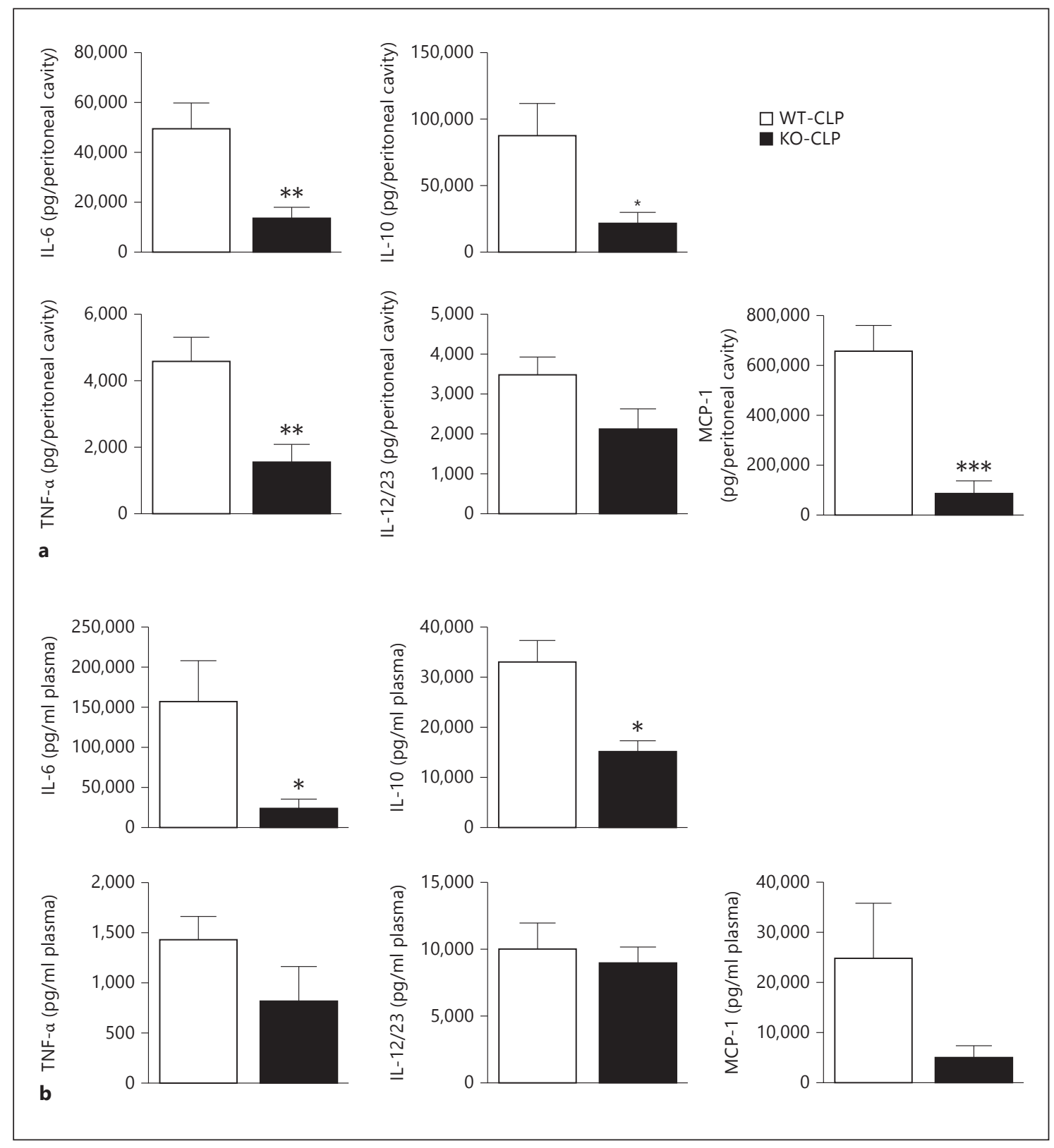

Fig. 1. GRK5 mediates inflammatory response in the CLP model of polymicrobial sepsis: GRK5 WT and KO mice were subjected to CLP surgery and peritoneal (a) and plasma (b) fluids were collected $12 \mathrm{~h}$ after surgery as described in Materials and Methods. IL-6, IL-10, TNFa, IL12/23 (total p40) and MCP-1 were measured in these two fluid samples using ELISA kits from eBiosciences $(n=12$ for WT and $n=9$ for KO for the 12-hour time point). ${ }^{*} \mathrm{p}<0.05,{ }^{* *} \mathrm{p}<0.01,{ }^{* * *} \mathrm{p}<0.001$ compared to the corresponding septic WT groups.

fer between the septic groups. Note that neither of the sham groups showed any detectable levels or showed very low levels of cytokines/chemokines at the time points tested (data not shown). Together, these results suggest that GRK5 mediates sepsis-induced local and systemic inflammation.

\section{GRK5 Mediates NFkB Signaling in Polymicrobial Sepsis}

Previous studies have shown that GRK5 is an important regulator of $\mathrm{NFKB}$ signaling and inflammatory gene expression in mouse, zebra fish and drosophila models $[14,19]$. Our results in the CLP model of polymicrobial 
sepsis suggest that GRK5 deficiency attenuates inflammatory cytokine production both in the peritoneal cavity and in the plasma. To examine if GRK5 deficiency leads to attenuated NFKB signaling in this sepsis model, we determined phospho-IкBa levels and mRNA expression of NFkB-dependent genes in the liver from the two genotypes of mice subjected to sham or CLP surgery. As shown in figure $2 \mathrm{a}$, CLP induced significant phosphorylation of IKBa in the liver of WT mice, and this was significantly inhibited in the GRK5 KO mice. To determine the expression of $\mathrm{NF} \kappa \mathrm{B}$-dependent genes, we examined the mRNA levels of I $\kappa a$, IL- 6 and IL- $1 \beta$ in the liver $12 \mathrm{~h}$ after CLP. Consistent with the role of GRK5 in IкBa phosphorylation, mRNA expression of $I \kappa B \alpha, I L-1 \beta$ and IL-6 was significantly inhibited in the GRK5 KO mice (fig. 2b). Interestingly, this phenomenon was also observed in the lungs (fig. 2b, lower panel). To rule out other signaling pathways, we also examined pERK, pJNK and pP38 and found no effect of GRK5 deficiency on these pathways (fig. 2c). Together, these results demonstrate a crucial role for GRK5 in NFKB activation in vivo and the consequent inflammatory response in this polymicrobial sepsis model.

GRK5 Does Not Regulate Chemotaxis to the Local Site of Injury/Infection

Studies have shown that following CLP, peritoneal cell infiltration plays a critical role in the progression of sepsis [33] and that modulation of immune cell infiltration can have therapeutic consequences [34]. GRKs have been shown to be important regulators of chemokine receptor signaling and chemotaxis [35]. Therefore, to determine if GRK5 regulates immune cell infiltration into the site of injury/infection (peritoneum), we examined the number of cells in the peritoneal cavity following CLP at different time points in the two genotypes of mice. As shown in figure 3, GRK5 deficiency did not affect immune cell infiltration into the peritoneal cavity at any of the time points tested. In addition, there was no difference in infiltration of specific immune cell populations (fig. 3). Together, these results suggest that GRK5 is an unlikely regulator of chemotaxis in this model of sepsis.

\section{Deficiency of GRK5 Does Not Affect Bacterial Load following Sepsis}

In order to examine if GRK5 is able to modulate bacterial load after CLP, we plated peritoneal lavage fluid and blood samples from sham and septic mice onto 5\% sheep blood agar plates and determined the colony-forming

GRK5 Mediates Sepsis-Induced Inflammation units. Interestingly, the bacterial load was not any different between the WT and the KO mice at any of the time points tested (fig. 4a, b). Taken together, our data so far suggest that even though GRK5 mediates NFKB signaling and inflammation in sepsis, neither chemotaxis nor bacterial load is significantly affected.

Regulation of Thymocyte Numbers by GRK5 in Sepsis

It is now well established that sepsis-induced thymocyte apoptosis contributes to the pathogenic events and the consequent mortality in septic animals [36] and human patients [37]. Importantly, inhibiting thymic apoptosis has been shown to be beneficial in preventing sepsisinduced mortality in experimental models [38]. Previous studies have suggested a role for GRK5 in irradiation-induced thymocyte apoptosis via a p53-dependent pathway [13]. Furthermore, NFKB signaling in thymocytes has been shown to be an important regulator of thymocyte apoptosis $[39,40]$. Together, based on these rationales, we hypothesized that GRK 5 could be an important regulator of sepsis-induced thymocyte apoptosis. To test this, we first assessed the number of thymocytes from WT and $\mathrm{KO}$ mice subjected to sham or CLP surgery. As predicted from previous studies, in the WT septic mice thymocyte numbers were significantly decreased compared to shamoperated mice (fig. 5a). Interestingly, however, septic KO mice had significantly higher numbers of thymocytes compared to the corresponding WT mice at both time points. In addition to the total cells, the frequency of double-positive CD4+CD8+ lymphocytes (loss of these lymphocytes has been linked to poor survival [41]) was also markedly decreased in the WT septic mice compared to the sham mice (fig. 5b). Importantly, the decrease in these double-positive $\mathrm{CD} 4+\mathrm{CD} 8+$ cells was significantly attenuated in the GRK5-deficient mice (fig. 5b).

Regulation of Thymocyte Apoptosis by GRK5 in vivo

To further confirm whether the difference in thymocyte numbers is the result of altered apoptosis between WT and GRK5 KO mice, we performed two separate assays: flow cytometry analysis of thymocytes stained with Annexin V and PI, and caspase activity assays. Consistent with the thymocyte numbers, an increased frequency of Annexin V+PI- cells (early apoptotic cells) was observed in WT septic mice, and this was significantly inhibited in the GRK5 KO mice (fig. 5c). Furthermore, CLP significantly induced caspase-3 activity in the WT thymocytes, and this was again markedly reduced in the GRK5 KO mice (fig. 5c). Unlike caspase-3, activities of caspase- 8 and 9 were not significantly induced in sepsis 

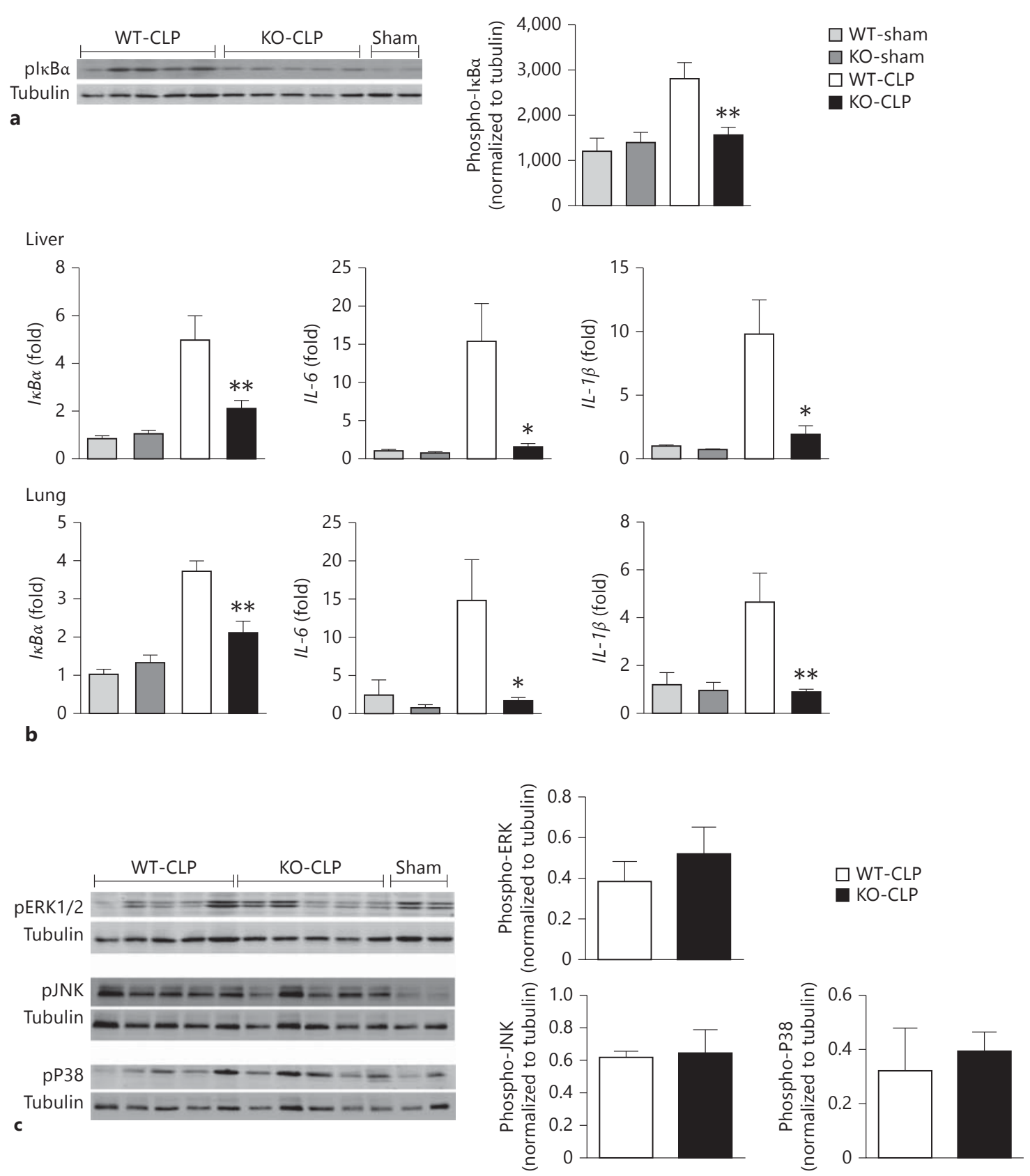

Fig. 2. GRK5 mediates I $\mathrm{B} \mathrm{B} \alpha$ phosphorylation and expression of NF- $\kappa B$-dependent genes in liver and lung. a Cytosolic extracts of the liver tissue from GRK5 WT and KO mice $12 \mathrm{~h}$ postsepsis were subjected to Western blotting for phospho-IкBa and tubulin (for normalization) as described before [14]. A representative blot is shown on the left and quantitation on the right $(\mathrm{n}=11-12$ each for $\mathrm{WT}$ and KO mouse CLP and $\mathrm{n}=3$ for shams; ${ }^{*} \mathrm{p}<0.05,{ }^{* *} \mathrm{p}<0.01$ ). b Liver and lung tissue samples from GRK5 WT and KO mice sub- jected to sham or CLP surgery were collected $12 \mathrm{~h}$ postsepsis and analyzed for the expression of NFKB-dependent genes as described in Materials and Methods $(n=8$ for CLP and $n=4$ for sham per genotype; ${ }^{*} \mathrm{p}<0.05,{ }^{* *} \mathrm{p}<0.01$ compared to the corresponding septic WT group). c Liver tissue extracts described in a were subjected to immunoblotting for pERK, pJNK and pP38 as described in Materials and Methods. Western blot is shown on the left and quantitation on the right ( $\mathrm{n}=5$ each for WT and KO mouse CLP) 
Fig. 3. Role of GRK5 in peritoneal cell infiltration following CLP: GRK5 WT and KO mice were subjected to CLP surgery, and peritoneal cells were collected by lavaging peritoneal cavity and were counted (a) as described in Materials and Methods. The different immune populations (b) were assessed by flow cytometry as described in Materials and Methods ( $\mathrm{n}=9$ for the 4 - and 12-hour time points and $n=5-6$ for the 20 and 36-hour time points, per genotype).
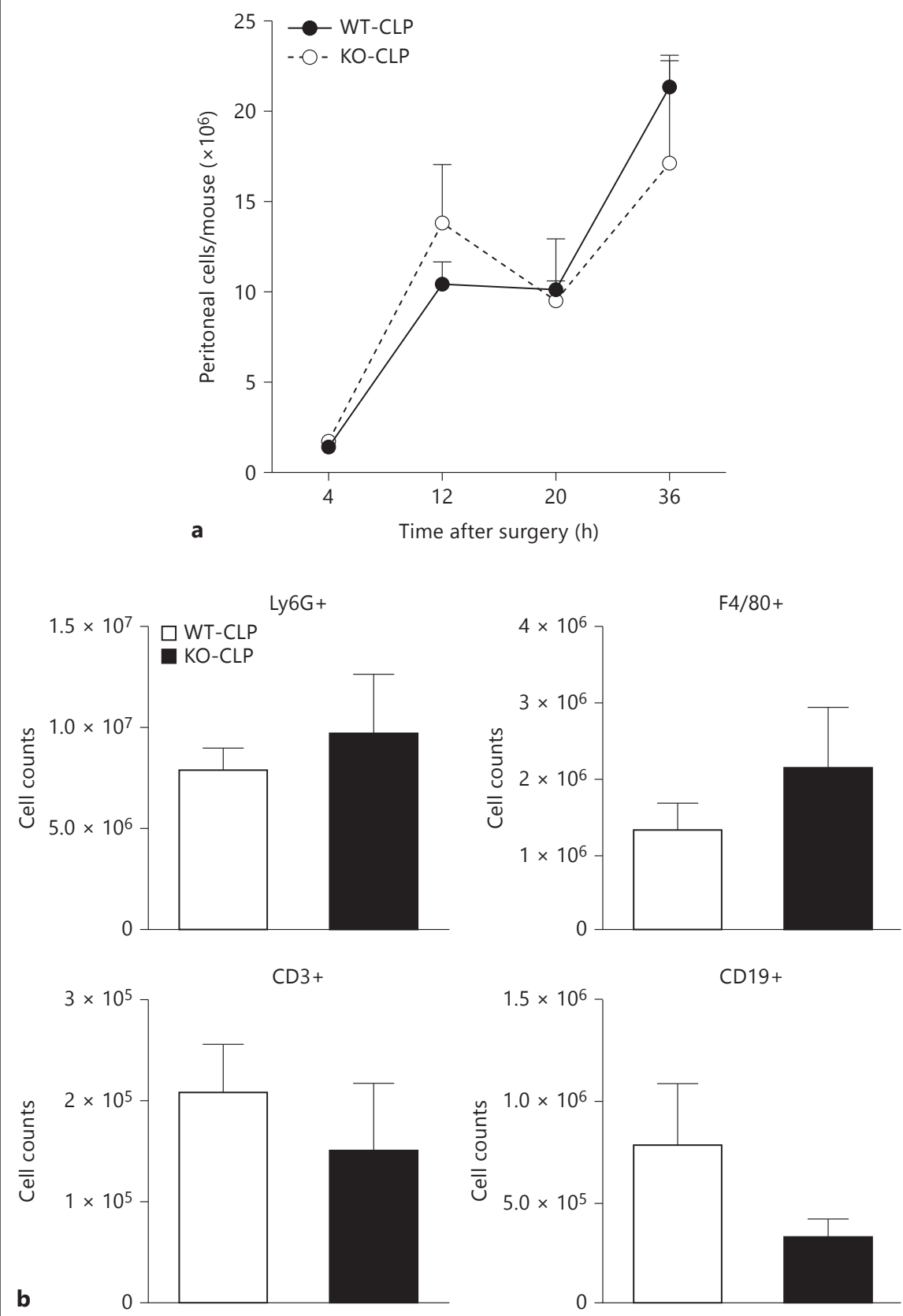

and did not differ between the groups (fig. 5c). Together, these results implicate GRK5 in the regulation of caspase-3-mediated thymocyte apoptosis following sepsis.

\section{Mechanism of GRK5-Mediated Thymic Apoptosis}

Previous studies have shown that sepsis-induced corticosteroids induce thymic apoptosis in the CLP model [42]. To determine whether GRK5 directly modulates cortico- steroid-induced thymocyte apoptosis, we stimulated thymocytes from WT and GRK5 KO mice ex vivo with dexamethasone (100 nM) and assessed apoptosis using flow cytometry (Annexin V/PI). Dexamethasone treatment induced significant apoptosis in both the genotypes and, surprisingly, apoptosis was equivalent between the genotypes (data not shown). Because these results ruled out any direct effect of GRK5 on thymic apoptosis (at least as in- 
Fig. 4. Role of GRK5 in bacterial load following CLP: blood and peritoneal samples from GRK5 WT and KO mice subjected to sham or CLP surgery were assessed for bacterial load as described in Materials and Methods. Colony-forming unit counts from peritoneal exudate (a) and blood (b) for different time points are shown from mice subjected to CLP ( $\mathrm{n}=11-14$ per genotype for the 12 -hour time point, $\mathrm{n}=5$ per genotype for the 20-hour time point and $n$ $=9-12$ for the 36-hour time point). Note that sham mice did not show any bacterial colonies (data not shown).
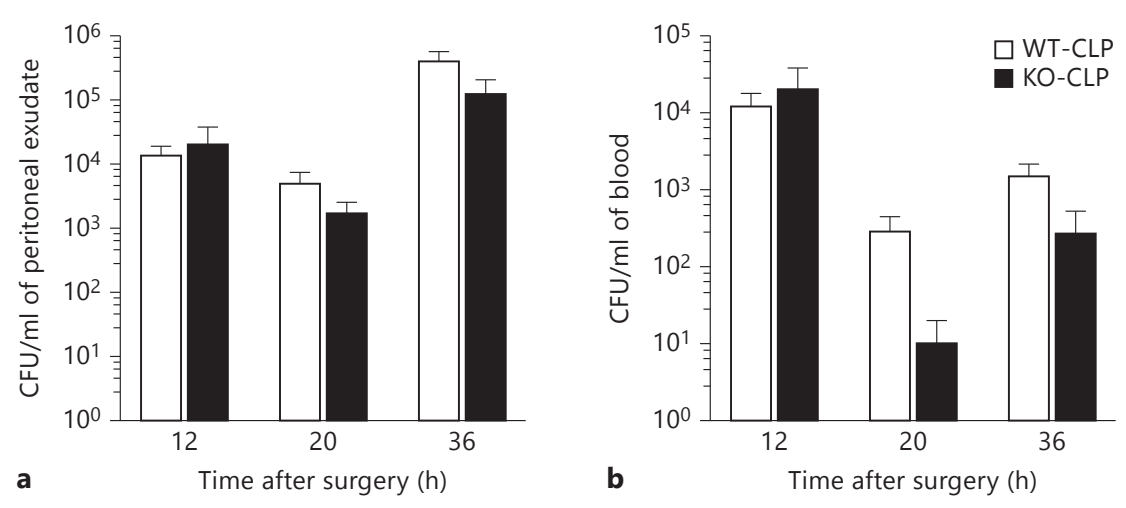

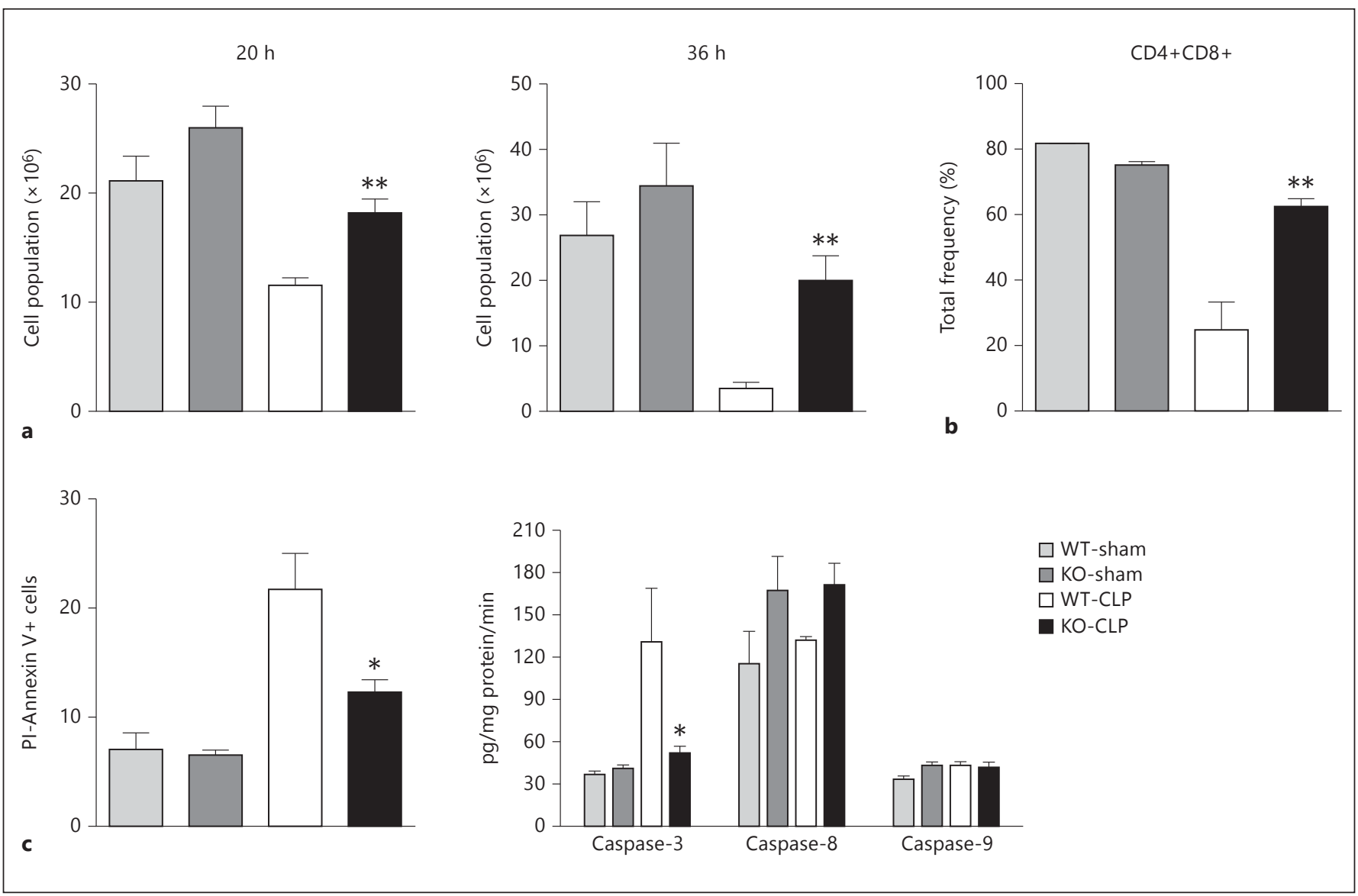

Fig. 5. GRK5 mediates thymocyte apoptosis in polymicrobial sepsis: thymi from GRK5 WT and KO mice subjected to sham or CLP surgery were collected 20 and $36 \mathrm{~h}$ after surgery as described in Materials and Methods. Thymocytes were analyzed for total number (a), CD4+CD8+ (b), Annexin V+ and PI- staining and for caspase activity (c) as described in Materials and Methods $(n=5-6$ per genotype for assessing total thymus cell number and Annexin $\mathrm{V}+$ and PI- staining and $\mathrm{n}=3-4$ per genotype for the flow cytometry experiments). ${ }^{*} \mathrm{p}<0.05,{ }^{* *} \mathrm{p}<0.01$ compared to the corresponding septic WT groups. 


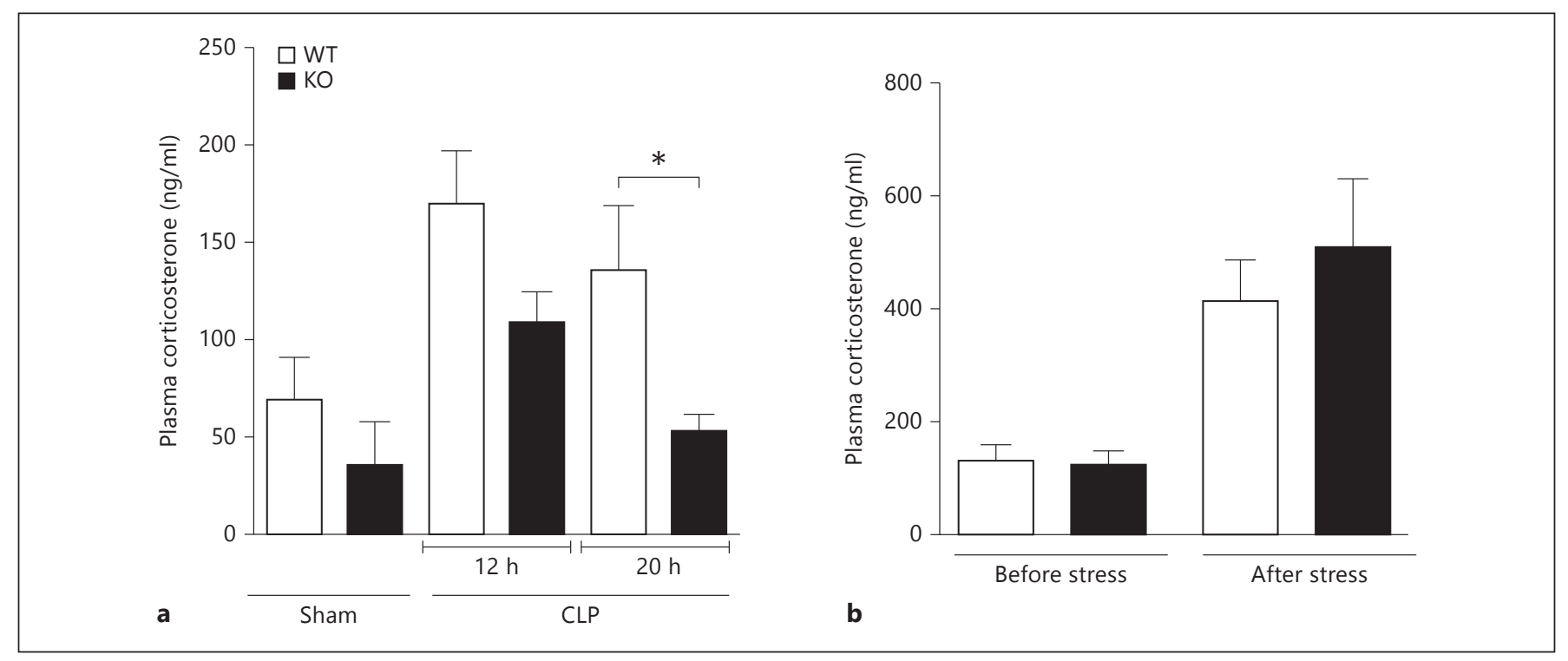

Fig. 6. Sepsis-induced plasma corticosterone levels are diminished in GRK5 KO mice. a Plasma samples collected 12 and $20 \mathrm{~h}$ after surgery (sham and CLP) from GRK5 WT and KO mice were assessed for corticosterone levels using the Cayman Chemical EIA kit ( $n=8$ per genotype for both the 12 - and 20-hour time point for

duced by corticosteroids), we next examined whether the levels of corticosterone are different between the two genotypes during sepsis progression. Interestingly, plasma corticosterone levels were significantly higher in the WT septic mice (compared to sham) and the levels were markedly attenuated in the GRK5 KO septic mice (fig. 6). This was more evident at the later time point $(20 \mathrm{~h})$. To determine whether the difference in the corticosterone levels between the two genotypes is specific to sepsis, we induced stress with physical restraint in both genotypes and measured plasma corticosterone levels. We found that $30 \mathrm{~min}$ of physical restraint induced a significant increase in plasma corticosterone, but the levels were similar between the two genotypes (fig. 6). Together, these results suggest that sepsis-induced corticosterone levels are attenuated in GRK5-deficient mice, and this might lead to enhanced thymocyte survival during later stages of sepsis.

\section{GRK5 Inhibits Immunoresponsiveness of Peritoneal \\ Cells in Sepsis}

Decrease in lymphocytes due to apoptosis is thought to be an important pathogenic event in the development of immunosuppression observed during sepsis progression. Because thymic apoptosis is reduced in the GRK5deficient mice, we hypothesized that the consequent development of immune suppression may be attenuated in
CLP and $\mathrm{n}=2-3$ for sham at the 12 -hour time point). $\mathbf{b}$ Corticosterone levels in plasma samples obtained from GRK5 WT and KO mice before and after restraint stress ( $\mathrm{n}=7$ per genotype). ${ }^{*} \mathrm{p}<$ 0.05 compared to the corresponding WT groups.

GRK5-deficient mice. To test this in vitro, we obtained peritoneal cells from the two genotypes of mice subjected to sepsis (36 h post-CLP) and assessed their immunocompetency in response to in vitro LPS stimulation. Supernatants from these experiments were assayed for IL-6, IL-10, TNFa and IL-12/23 using ELISA. As expected, unstimulated GRK5 KO cells (from septic mice) produced lower cytokine levels compared to the WT septic mice (fig. 7). However, upon stimulation with LPS, GRK5 KO cells produced significantly enhanced proinflammatory cytokines (IL-6, TNFa and IL-12/23) compared to the WT cells. This effect was restricted only to the typical proinflammatory group and not to IL-10. The basal IL-10 level was much higher in the WT cells from septic mice and LPS stimulation did not further enhance IL-10. Together, these results show that even though the initial inflammatory response in the KO cells is attenuated, cells from these septic mice respond better than the WT cells to in vitro LPS stimulation. This suggests that deficiency of GRK5 possibly renders the mice more immune-responsive at later stages of sepsis.

\section{Role of GRK5 in Sepsis-Induced Mortality}

Dysregulated inflammatory response, poor bacterial clearance and excessive loss of lymphocytes have all been linked to poor survival in sepsis [21]. Our results using 
Fig. 7. GRK5 suppresses immunoresponsiveness of peritoneal cells in sepsis: peritoneal cells from GRK5 WT and KO mice subjected to sham or CLP surgery were collected $36 \mathrm{~h}$ postsepsis, stimulated with LPS and assessed for cytokine production as described in Materials and Methods $(\mathrm{n}=7-8$ per genotype). ${ }^{*} \mathrm{p}<0.05,{ }^{* *} \mathrm{p}<0.01 \mathrm{com}-$ pared to the corresponding septic WT peritoneal cells stimulated with LPS; ${ }^{*} \mathrm{p}<0.05$ compared to the corresponding septic WT peritoneal cells without LPS stimulation.

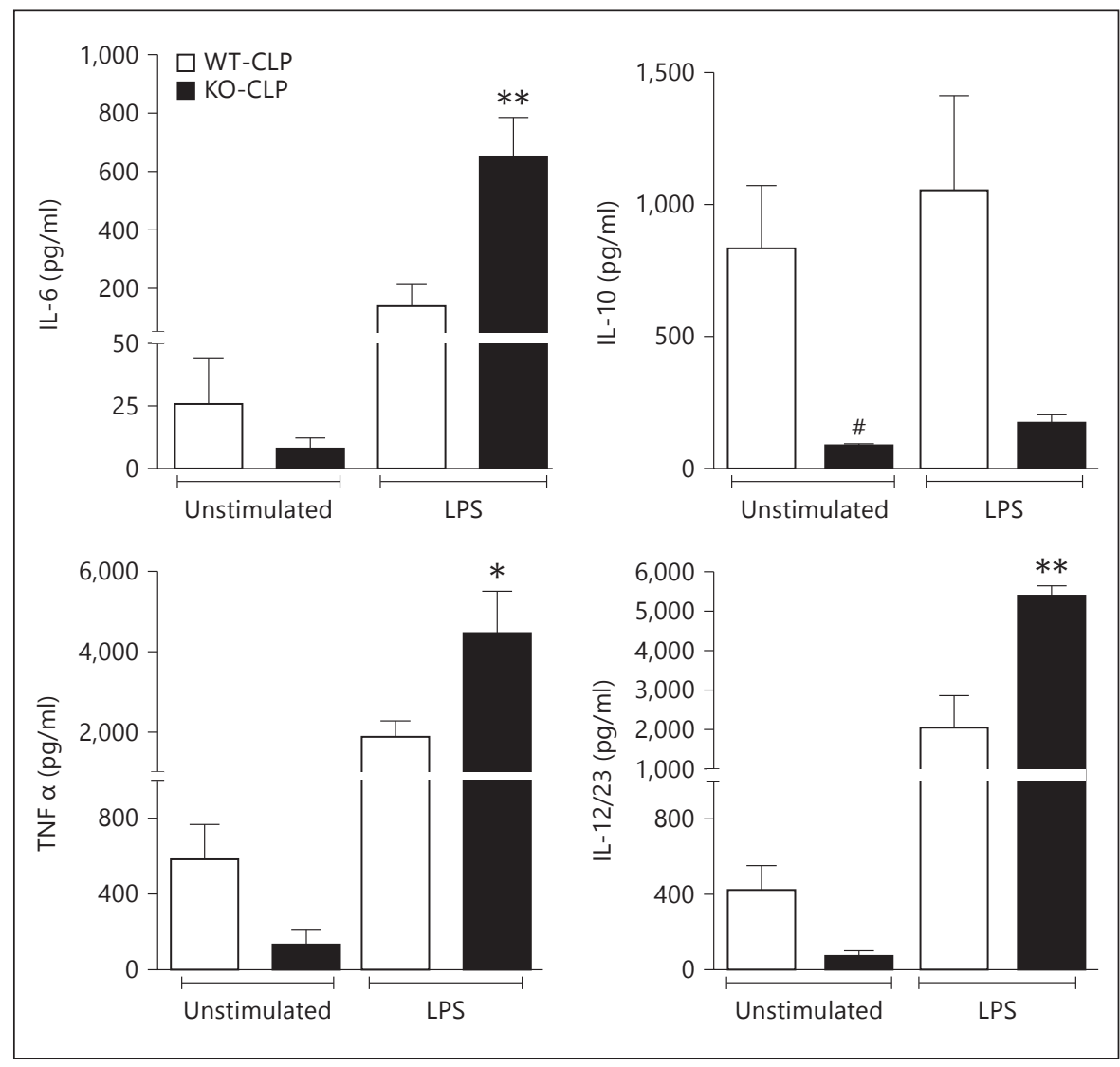

Fig. 8. Role of GRK5 on survival following CLP: GRK5 WT and KO mice were subjected to sham or CLP surgery and survival was assessed for 7 days in the absence (a) or presence (b) of antibiotics as described in Materials and Methods ( $\mathrm{n}=10$ per genotype in $\mathbf{a} ; \mathrm{n}=15-16$ per genotype in $\mathbf{b}$ ). ${ }^{*} \mathrm{p}$ $<0.05$ compared to the corresponding septic WT in the antibiotic-treated group by log-rank test. Factorial design analysis of the data showed the following: between genotypes: $p=0.204$, between antibiotic and nonantibiotic groups: $\mathrm{p}=0.024$, and interaction between genotypes and with antibiotics: $\mathrm{p}=0.038$.

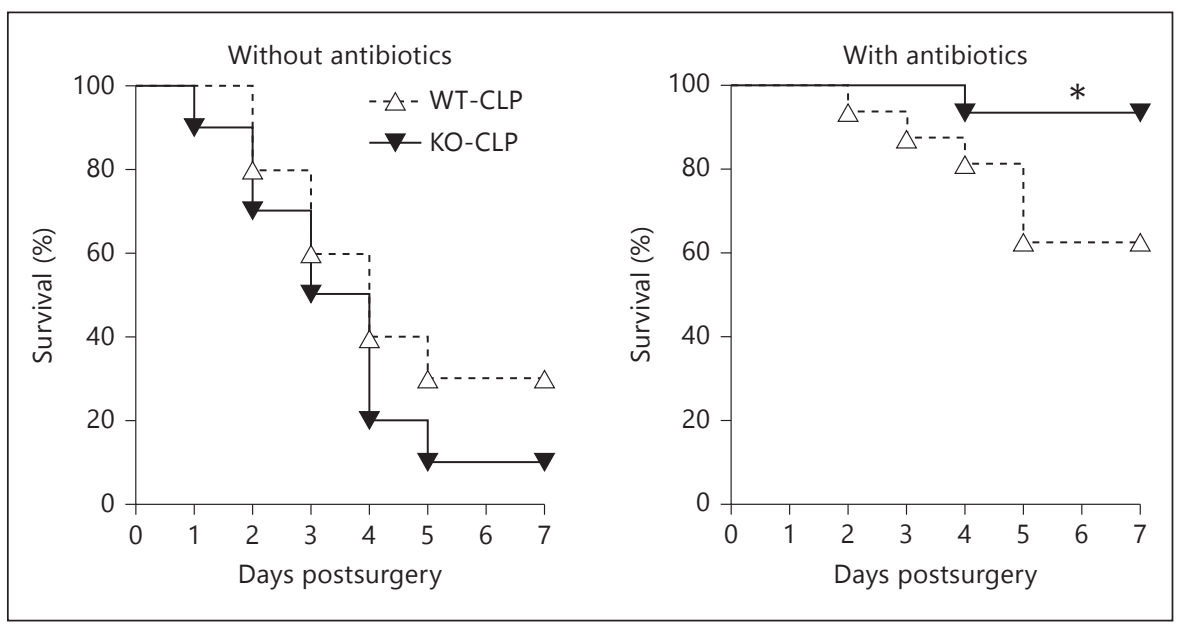

GRK5-deficient mice indicate that GRK5 mediates inflammatory response and thymocyte apoptosis in sepsis. Therefore, we predicted that septic GRK5-deficient mice might exhibit altered survival profile compared to the corresponding WT mice. Contrary to our expectations, mortality following sepsis was similar between the two genotypes (fig. 8a). We then reasoned that because bacterial load was similar between the two genotypes, GRK5deficient mice might exhibit better survival in the presence of antibiotics. To test this, we subjected WT and 
GRK5-deficient mice to CLP and administered antibiotics (ceftriaxone: $25 \mu \mathrm{g} / \mathrm{g}$ body weight and metronidazole: $15 \mu \mathrm{g} / \mathrm{g}$ body weight) intraperitoneally $1 \mathrm{~h}$ post-CLP and every $24 \mathrm{~h}$ thereafter for 5 days [43]. Compared to WT mice that did not receive any antibiotics ( $\sim 70 \%$ mortality), mortality in WT mice that received antibiotics decreased to $\sim 40 \%$. Interestingly, however, GRK5-deficient mice receiving antibiotics had only $\sim 10 \%$ mortality (compared to $\sim 90 \%$ mortality in GRK5-deficient mice not receiving antibiotics) (fig. 8b). Together, these results demonstrate that in the presence of antibiotics, GRK5 deficiency protects mice from sepsis-induced mortality.

\section{Discussion}

Given the high rate of mortality in sepsis, understanding the pathophysiologic events and molecular mechanisms that mediate mortality in sepsis can help in developing new therapies and better treatment strategies. In the CLP model of polymicrobial sepsis GRK5 deficiency inhibited several but not all aspects of sepsis progression. Importantly, GRK5 deficiency significantly enhanced survival only in the presence of antibiotics. Even though GRK5 deficiency attenuated the inflammatory response both at the systemic and organ levels, as well as significantly inhibited thymocyte apoptosis, these changes were not sufficient to enhance survival. It is possible that both the severity of sepsis and bacterial dissemination were too high, in spite of the favorable effects on inflammation and thymocyte apoptosis in GRK5 deficiency. This is in part supported by the observation that the bacterial load per se was not different between the two genotypes of mice at early or later time points of sepsis. Thus it is possible that at this level of severity, both antibiotics (to clear bacterial infection) and GRK5 deficiency yield a better outcome. A similar phenotype was reported in MyD88 KO mice [44] wherein deficiency of MyD88 led to a diminished inflammatory response and attenuated lymphocyte apoptosis without any effect on bacterial load following polymicrobial sepsis. Importantly, similar to our studies, these effects were not sufficient to prevent mortality following CLP. MyD88 is a critical adaptor molecule for many TLRs including TLR4. Consistent with the phenotype of MyD88 KO mice, blocking TLR4 alone in a CLP model of sepsis did not prevent mortality because of persistent bacterial load [32]. Similar to our model, the TLR4 antagonist significantly improved survival only in the presence of antibiotics [32]. Based on our previous studies [14, 18] showing that GRK5 is an important regulator of TLR4 signal-

GRK5 Mediates Sepsis-Induced Inflammation ing, our results are consistent with these other studies in terms of the outcome of sepsis.

Studies have consistently found that the cytokine response plays a major role in the resolution of sepsis by the activation of immune cells and subsequent clearance of microbes. However, an excessive production of cytokines with aberrant activation of immune cells can have ill effects on the host. As shown in the endotoxemia model [14], we demonstrate here that GRK5 deficiency attenuates inflammatory cytokines following intra-abdominal polymicrobial sepsis. Consistent with the systemic and peritoneal cytokines, mRNA expression of NFkBdependent genes in the liver and lungs were also attenuated at $12 \mathrm{~h}$ postsepsis. In line with our observations in the LPS model as well as the demonstrated effects on bacterial infection in drosophila and zebra fish models, polymicrobial sepsis-induced IкBa phosphorylation in the liver was significantly inhibited in the GRK5 KO mice. Together, these studies are consistent with previous studies showing that GRK5 regulates NFKB signaling and therefore is able to modulate NFkB-dependent gene expression.

Contrary to our results in mice and those of Valanne et al. [19] in drosophila, zebra fish and human cells, other studies have also shown that GRK5 is a negative regulator of NFKB signaling in endothelial cells [17] and vascular smooth muscle cells [17]. This effect of GRK5 has been linked to its role in stabilizing nuclear I $\kappa \mathrm{B} \alpha$ levels whereas we have demonstrated previously that GRK5 is able to phosphorylate IKBa at the same sites as that of IKK $\beta$ and to mediate degradation [9]. Thus it is possible that GRK5 may have multiple roles in terms of $\mathrm{NFKB}$ regulation but the dominance of regulation might depend on the cell type and disease being examined.

Lymphocyte apoptosis has increasingly been recognized as an important step in the pathogenesis of sepsis, by inducing a state of 'immune paralysis' that renders the host vulnerable to invading pathogens [41]. NFKB signaling plays a vital role in lymphocyte development, function and apoptosis. In addition, NFKB signaling can either promote survival [39] or apoptosis [40] of lymphocytes depending on the cell type involved. Even though we found GRK5 to be an important regulator of NFKB signaling in the liver, this phenomenon appeared to be tissue specific since we did not observe any difference in $\mathrm{NF \kappa B}$ activity in the thymus from the two genotypes subjected to CLP (data not shown). Since previous studies have shown that sepsis-induced thymocyte apoptosis is mediated by corticosterone [42], we hypothesized that either corticosteroid signaling or its plasma levels may be dif- 
ferentially regulated in the two genotypes. Even though our in vitro results rule out any direct effect of GRK5 on corticosteroid-induced thymocyte apoptosis, our in vivo results demonstrate that GRK5 is an important regulator of sepsis-mediated increase in corticosterone levels. Since sepsis-induced thymocyte apoptosis has been shown to be mediated by corticosterone, lower levels of plasma corticosterone may in part explain why GRK5 KO thymocytes are protected from apoptosis in vivo. In future studies, we will determine whether the effect of GRK5 on thymocyte apoptosis in vivo is due to decreased corticosterone levels or due to differential regulation of other signals that may be involved in thymocyte apoptosis in sepsis.

Consistent with the better survival of thymocytes in the GRK5-deficient mice, septic peritoneal cells from the GRK5 KO were more immunocompetent than the cells from WT mice. While our studies do not demonstrate a cause-effect relationship between thymocyte apoptosis and peritoneal cell responsiveness in the septic mice, previous studies have suggested that enhanced lymphocyte apoptosis leads to enhanced immunosuppression in animal models [45]. In addition, it is possible that enhanced plasma corticosterone levels in the WT mice might suppress LPS-induced cytokine production in the WT cells compared to less suppression in the KO cells [46]. These mechanisms will be dissected out in future studies. Regardless of the mechanism, our results suggest that under conditions where the innate cells are already exposed to polymicrobial injury, GRK5 deficiency is able to modulate the cells to be more immune-responsive compared to the WT. The better immunoresponsiveness of innate cells during the stage of sepsis when cells are likely 'paralyzed' may lead to a beneficial outcome in sepsis [47]. This has been shown before where innate immune paralysis observed later in sepsis has been attributed to poor outcome [48]. Together, these results suggest that GRK5 deficiency could modulate sepsis at several levels or stages.

In conclusion, we demonstrate here that GRK5 is an important regulator of sepsis pathogenesis and that blocking GRK5 could result in a protective mechanism via modulating several aspects of sepsis. Even though bacterial load is not affected by GRK5, administration of antibiotics in the presence of GRK5 inhibition might lead to a better outcome in sepsis-induced mortality.

\section{Acknowledgements}

We gratefully acknowledge the support from NIH (grants HL095637, AR055726 and AR056680 to N.P.). We thank the University Lab Animal Resources for taking excellent care of our animals, the Histopathology Laboratory for their excellent service and Mr. Vernon LaLone for excellent technical assistance. We also thank the Michigan State University's Center for Statistical Training and Consulting for their consultation with regard to the survival analysis.

\section{References}

1 Premont RT, Gainetdinov RR: Physiological roles of $\mathrm{G}$ protein-coupled receptor kinases and arrestins. Annu Rev Physiol 2007;69: 511-534.

2 Gurevich EV, Tesmer JJ, Mushegian A, Gurevich VV: G protein-coupled receptor kinases: more than just kinases and not only for GPCRs. Pharmacol Ther 2012;133:40-69.

-3 Kunapuli P, Onorato JJ, Hosey MM, Benovic JL: Expression, purification, and characterization of the $\mathrm{G}$ protein-coupled receptor kinase GRK5. J Biol Chem 1994;269:1099-1105.

4 Gainetdinov RR, Bohn LM, Walker JK, Laporte SA, Macrae AD, Caron MG, Lefkowitz RJ, Premont RT: Muscarinic supersensitivity and impaired receptor desensitization in $\mathrm{G}$ protein-coupled receptor kinase 5-deficient mice. Neuron 1999;24:1029-1036.
5 Barthet G, Carrat G, Cassier E, Barker B, Gaven F, Pillot M, Framery B, Pellissier LP, Augier J, Kang DS, Claeysen S, Reiter E, Baneres JL, Benovic JL, Marin P, Bockaert J, Dumuis A: Betaarrestin1 phosphorylation by GRK5 regulates G protein-independent 5-HT4 receptor signalling. EMBO J 2009;28:2706-2718.

6 Chen Y, Wang F, Long H, Wu Z, Ma L: GRK5 promotes $\mathrm{F}$-actin bundling and targets bundles to membrane structures to control neuronal morphogenesis. J Cell Biol 2011;194: 905-920.

7 Martini JS, Raake P, Vinge LE, DeGeorge BR Jr, Chuprun JK, Harris DM, Gao E, Eckhart AD, Pitcher JA, Koch WJ: Uncovering G protein-coupled receptor kinase-5 as a histone deacetylase kinase in the nucleus of cardiomyocytes. Proc Natl Acad Sci USA 2008;105: 12457-12462.

8 Barker BL, Benovic JL: G protein-coupled receptor kinase 5 phosphorylation of hip regulates internalization of the chemokine receptor CXCR4. Biochemistry 2011;50:6933-6941.
9 Patial S, Luo J, Porter KJ, Benovic JL, Parameswaran N: G-protein-coupled-receptor kinases mediate TNFalpha-induced NFkappaB signalling via direct interaction with and phosphorylation of IkappaBalpha. Biochem J 2010; 425:169-178.

10 Parameswaran N, Pao CS, Leonhard KS, Kang DS, Kratz M, Ley SC, Benovic JL: Arrestin-2 and $G$ protein-coupled receptor kinase 5 interact with NFkappaB1 p105 and negatively regulate lipopolysaccharide-stimulated ERK1/2 activation in macrophages. J Biol Chem 2006; 281:34159-34170.

11 Niehrs C, Shen J: Regulation of Lrp6 phosphorylation. Cell Mol Life Sci 2010;67:2551-2562.

-12 So CH, Michal AM, Mashayekhi R, Benovic JL: G protein-coupled receptor kinase 5 phosphorylates nucleophosmin and regulates cell sensitivity to polo-like kinase 1 inhibition. J Biol Chem 2012;287:17088-17099. 
13 Chen X, Zhu H, Yuan M, Fu J, Zhou Y, Ma L: G-protein-coupled receptor kinase 5 phosphorylates p53 and inhibits DNA damage-induced apoptosis. J Biol Chem 2010;285:1282312830.

14 Patial S, Shahi S, Saini Y, Lee T, Packiriswamy N, Appledorn DM, Lapres JJ, Amalfitano A, Parameswaran N: G-protein coupled receptor kinase 5 mediates lipopolysaccharide-induced NFkappaB activation in primary macrophages and modulates inflammation in vivo in mice. J Cell Physiol 2011;226:1323-1333.

15 Kim JI, Chakraborty P, Wang Z, Daaka Y: Gprotein coupled receptor kinase 5 regulates prostate tumor growth. J Urol 2012;187:322329.

16 Cheng S, Li L, He S, Liu J, Sun Y, He M, Grasing K, Premont RT, Suo WZ: GRK5 deficiency accelerates \{beta\}-amyloid accumulation in Tg2576 mice via impaired cholinergic activity. J Biol Chem 2010;285:41541-41548.

-17 Wu JH, Zhang L, Fanaroff AC, Cai X, Sharma KC, Brian L, Exum ST, Shenoy SK, Peppel K, Freedman NJ: G protein-coupled receptor kinase- 5 attenuates atherosclerosis by regulating receptor tyrosine kinases and 7-transmembrane receptors. Arterioscler Thromb Vasc Biol 2012;32:308-316.

-18 Packiriswamy N, Parvataneni S, Parameswaran N: Overlapping and distinct roles of GRK5 in TLR2-, and TLR3-induced inflammatory response in vivo. Cell Immunol 2012;272: 107-111.

19 Valanne S, Myllymaki H, Kallio J, Schmid MR, Kleino A, Murumagi A, Airaksinen L, Kotipelto T, Kaustio M, Ulvila J, Esfahani SS, Engstrom Y, Silvennoinen O, Hultmark D, Parikka M, Ramet M: Genome-wide RNA interference in drosophila cells identifies G protein-coupled receptor kinase 2 as a conserved regulator of NF-kappaB signaling. J Immunol 2010;184:6188-6198.

20 Hall MJ, Williams SN, DeFrances CJ, Golosinskiy A: Inpatient care for septicemia or sepsis: a challenge for patients and hospitals. NCHS Data Brief 2011:1-8.

21 Stearns-Kurosawa DJ, Osuchowski MF, Valentine C, Kurosawa S, Remick DG: The pathogenesis of sepsis. Annu Rev Pathol 2011; 6:19-48.

22 Remick DG, Ward PA: Evaluation of endotoxin models for the study of sepsis. Shock 2005;24(suppl 1):7-11.

-23 Hubbard WJ, Choudhry M, Schwacha MG, Kerby JD, Rue LW 3rd, Bland KI, Chaudry IH: Cecal ligation and puncture. Shock 2005; 24(suppl 1):52-57.

-24 Parvataneni S, Gonipeta B, Packiriswamy N, Lee T, Durairaj H, Parameswaran N: Role of myeloid-specific G-protein coupled receptor kinase-2 in sepsis. Int J Clin Exp Med 2011;4: 320-330.
25 Porter KJ, Gonipeta B, Parvataneni S, Appledorn DM, Patial S, Sharma D, Gangur V, Amalfitano A, Parameswaran N: Regulation of lipopolysaccharide-induced inflammatory response and endotoxemia by beta-arrestins. J Cell Physiol 2010;225:406-416.

26 Groesdonk HV, Wagner F, Hoffarth B, Georgieff $M$, Senftleben U: Enhancement of NFkappaB activation in lymphocytes prevents $\mathrm{T}$ cell apoptosis and improves survival in murine sepsis. J Immunol 2007;179:8083-8089.

27 Shounan Y, Feng X, O'Connell PJ: Apoptosis detection by annexin $\mathrm{V}$ binding: a novel method for the quantitation of cell-mediated cytotoxicity. J Immunol Methods 1998;217: 61-70.

28 Kusner LL, Sarthy VP, Mohr S: Nuclear translocation of glyceraldehyde-3-phosphate dehydrogenase: a role in high glucose-induced apoptosis in retinal muller cells. Invest Ophthalmol Vis Sci 2004;45:1553-1561.

29 Nair A, Bonneau RH: Stress-induced elevation of glucocorticoids increases microglia proliferation through NMDA receptor activation. J Neuroimmunol 2006;171:72-85.

30 Leelahavanichkul A, Bocharov AV, Kurlander R, Baranova IN, Vishnyakova TG, Souza AC, Hu X, Doi K, Vaisman B, Amar M, Sviridov D, Chen Z, Remaley AT, Csako G, Patterson AP, Yuen PS, Star RA, Eggerman TL: Class B scavenger receptor types I and II and CD36 targeting improves sepsis survival and acute outcomes in mice. J Immunol 2012;188: 2749-2758.

31 Romero CR, Herzig DS, Etogo A, Nunez J, Mahmoudizad R, Fang G, Murphey ED, Toliver-Kinsky T, Sherwood ER: The role of interferon-gamma in the pathogenesis of acute intra-abdominal sepsis. J Leukoc Biol 2010; 88:725-735.

32 Sha T, Iizawa Y, Ii M: Combination of imipenem and TAK-242, a toll-like receptor 4 signal transduction inhibitor, improves survival in a murine model of polymicrobial sepsis. Shock 2011;35:205-209.

33 Craciun FL, Schuller ER, Remick DG: Early enhanced local neutrophil recruitment in peritonitis-induced sepsis improves bacterial clearance and survival. J Immunol 2010;185: 6930-6938.

- 34 He M, Moochhala SM, Adhikari S, Bhatia M: Administration of exogenous fractalkine, a CX3C chemokine, is capable of modulating inflammatory response in cecal ligation and puncture-induced sepsis. Shock 2009;31:3339.

-35 Arraes SM, Freitas MS, da Silva SV, de Paula Neto HA, Alves-Filho JC, Auxiliadora Martins M, Basile-Filho A, Tavares-Murta BM, Barja-Fidalgo C, Cunha FQ: Impaired neutrophil chemotaxis in sepsis associates with GRK expression and inhibition of actin assembly and tyrosine phosphorylation. Blood 2006;108:2906-2913.

-36 Wang SD, Huang KJ, Lin YS, Lei HY: Sepsisinduced apoptosis of the thymocytes in mice. J Immunol 1994;152:5014-5021.
37 Hotchkiss RS, Tinsley KW, Swanson PE, Schmieg RE Jr, Hui JJ, Chang KC, Osborne DF, Freeman BD, Cobb JP, Buchman TG, Karl IE: Sepsis-induced apoptosis causes progressive profound depletion of B and CD4+ T lymphocytes in humans. J Immunol 2001; 166:6952-6963.

38 Weaver JG, Rouse MS, Steckelberg JM, Badley $\mathrm{AD}$ : Improved survival in experimental sepsis with an orally administered inhibitor of apoptosis. FASEB J 2004; 18:1185-1191.

39 Beg AA, Baltimore D: An essential role for NF-kappaB in preventing TNF-alpha-induced cell death. Science 1996;274:782-784.

40 Bessho R, Matsubara K, Kubota M, Kuwakado K, Hirota H, Wakazono Y, Lin YW, Okuda A, Kawai M, Nishikomori R, et al.: Pyrrolidine dithiocarbamate, a potent inhibitor of nuclear factor kappa B (NF-kappa B) activation, prevents apoptosis in human promyelocytic leukemia HL-60 cells and thymocytes. Biochem Pharmacol 1994;48:1883-1889.

41 Hotchkiss RS, Tinsley KW, Swanson PE, Chang KC, Cobb JP, Buchman TG, Korsmeyer SJ, Karl IE: Prevention of lymphocyte cell death in sepsis improves survival in mice. Proc Natl Acad Sci USA 1999;96:1454114546.

42 Ayala A, Herdon CD, Lehman DL, DeMaso CM, Ayala CA, Chaudry IH: The induction of accelerated thymic programmed cell death during polymicrobial sepsis: Control by corticosteroids but not tumor necrosis factor. Shock 1995;3:259-267.

43 Turnbull IR, Wlzorek JJ, Osborne D, Hotchkiss RS, Coopersmith CM, Buchman TG: Effects of age on mortality and antibiotic efficacy in cecal ligation and puncture. Shock 2003;19:310-313.

44 Peck-Palmer OM, Unsinger J, Chang KC, Davis CG, McDunn JE, Hotchkiss RS: Deletion of MyD88 markedly attenuates sepsis-induced T and B lymphocyte apoptosis but worsens survival. J Leukoc Biol 2008;83:1009-1018.

45 Inoue S, Unsinger J, Davis CG, Muenzer JT, Ferguson TA, Chang K, Osborne DF, Clark AT, Coopersmith CM, McDunn JE, Hotchkiss RS: IL-15 prevents apoptosis, reverses innate and adaptive immune dysfunction, and improves survival in sepsis. J Immunol 2010; 184:1401-1409.

46 Wang X, Nelin LD, Kuhlman JR, Meng X, Welty SE, Liu Y: The role of map kinase phosphatase- 1 in the protective mechanism of dexamethasone against endotoxemia. Life Sci 2008;83:671-680.

47 Ellaban E, Bolgos G, Remick D: Selective macrophage suppression during sepsis. Cell Immunol 2004;231:103-111.

48 Guo RF, Riedemann NC, Ward PA: Role of C5a-C5aR interaction in sepsis. Shock 2004; 21:1-7.
GRK5 Mediates Sepsis-Induced Inflammation
J Innate Immun 2013;5:401-413 DOI: $10.1159 / 000347002$ 